\title{
SINTOMATOLOGIA INTESTINAL NA FASE CRÔNICA DA ESQUISTOSSOMOSE MANSONI
}

\author{
Eleonora L. Peixinho, Sumaia B. André e \\ José Carlos Bina
}

\begin{abstract}
Com o objetivo de investigar os sintomas intestinais na fase crônica da esquistossomose mansoni, estudamos 102 individuos na faixa etária de nove a dezenove anos, dos 144 previamente selecionados entre os moradores autóctones dos distritos de Lagoa Redonda e Menezes, portadores de Schistosoma mansoni. Todos foram submetidos a uma anamnese segmentar com a finalidade de detectar a presença de cinco sintomas intestinais: dor abdominal, diarréia, muco e rajas de sangue nas fezes e enterorragia. Metade dos pacientes foi medicada com mebendazole e posteriormente com oxamniquine, constituindo-se no grupo caso, enquanto a outra metade, tratada apenas com mebendazole, constituiu-se no grupo controle. Os resultados mostram que a esquistossomose é provavelmente a responsável pelas queixas de muco e/ou rajas de sangue nas fezes, ocorrendo uma reducão dessas queixas, estatisticamente significante, após a terapêutica especifica.
\end{abstract}

Palavras-chaves: Schistosoma mansoni. Esquistossomose. Fase crônica da esquistossomose. Sintomas intestinais.

As investigações realizadas com o objetivo de determinar os sintomas produzidos pela esquistossomose mansoni, encontraram sempre dificuldades para estabelecer relaçào de causa e efeito. devido à falta de controle de outros fatores contribuintes, como as associaçōes com outras parasitoses ou condiçoes patologicas.

Sintomatologia bastante diversificada foi descrita por vários autores, porém em trabalhos não controlados adequadamente1 378912 . Em 1966 Barbosa ${ }^{1}$ em estudos sobre a morbidade da esquistossomose em quatro localidades de Pernambuco, chamou a atenção para a falta de peculiaridade dos sintomas dessa doença. Em 1976, Conceição ${ }^{3}$ e Menezes7, trabalhando em duas diferentes localidades rurais, não observaram diferenças dos sintomas gastrointestinais entre os pacientes infectados e não infectados, exceto quanto à presença de enterorragia.

O reduzido número de trabalhos visando caracterizar os sintomas clínicos causados por essa parasitose, foi a principal motivação na realização do presente trabalho, que tem por objetivo o estudo da sintomatologia intestinal na fase crônica da esquistossomose mansoni.

\section{MATERIAL E MÉTODOS}

O material para realização do presente trabalho é constituido de parte das populações de Lagoa

Faculdade de Medicina da Universidade Federal da Bahia e Centro de Pesquisas Gonçalo Moniz (FIOCRUZ).

Endereço para correspondência: Rua Guadalajara, 430/902

- Morro do Gato - 40000 Salvador-BA - Brasil.

Recebido para publicação em 27/8/84.
Redonda e Menezes, distritos do Municipio de Sapeaçu-Bahia, localizado na zona fisiográfica do Recôncavo Baiano, distando cerca de $200 \mathrm{Km}$ de Salvador. Nas duas áreas existem coleções hidricas com vegetação aquática onde são encontrados caramujos do gênero Biomphalaria - glabrata e straminea; a população serve-se dessa água para banhos e usos domésticos. A economia da regiào está fundamentada no cultivo de fumo, mandioca e laranja.

A amostra foi constituida de moradores autóctones dos distritos de Lagoa Redonda e Menezes, portadores de Schistosoma mansoni, de ambos os sexos, na faixa etária de nove a dezenove anos, presentes nas duas áreas por ocasião do inquérito. Apesar de se ter planejado incluir todos os individuos na faixa etária determinada, isto é, 73 individuos do grupo caso e 71 do grupo controle, as dificuldades na localização dos pacientes fizeram com que fossem incluídos todos aqueles que preenchiam os requisitos na área de Menezes, no dia estabelecido para o tratamento, num total de 51 indivíduos, e os primeiros 51 individuos encontrados em Lagoa Redonda, distrito em que as habitaçōes são distantes e de dificil acesso.

O diagnóstico das helmintoses foi feito através do exame de fezes qualitativo pelo método da sedimentação espontânea, e a intensidade da infecção pelo $S$. mansoni foi avaliada atraves de exame de fezes quantitativo pelo método de Kato \& Miura, modificado por Katz, Chaves e Pellegrino 4 .

Todos os indivíduos da amostra foram submetidos a uma anamnese e exame físico da região abdominal. A anamnese incluiu a investigação de 
cinco sintomas intestinais: diarréia, enterorragia, fezes com muco, fezes com rajas de sangue e dor abdominal.

O conceito aplicado para diarréia foi a eliminação de fezes amolecidas ou fluidas, independentemente do número de dejeções diárias; enterorragia foi definida como perda sangüinea de coloração rutilante, eliminada através do ânus, sem associação com a matéria fecal; dor abdominal era investigada como presente ou ausente, independentemente de suas caracteristicas.

O exame físico do abdômen foi realizado com o paciente em decúbito dorsal e a palpação do figado $e$ do baço obedecia a seguinte metodologia: o tamanho dos órgãos medidos em centímetros, desde que ultrapassassem o rebordo costal sem manobras respiratórias. Quando este evento não ocorria, registrava-se que o órgào era impalpável, palpável à inspiração profunda ou palpável ao nivel do rebordo costal. Em relação ao figado as medidas foram feitas no rebordo costal perpendicular à linha médio-clavicular e no apêndice xifóide, visando individualizar os lobos direito e esquerdo. Todos os pacientes foram examinados pelo mesmo investigador (E.L.P) e os sintomas foram exclusivamente obtidos dos pacientes, não sendo valorizados os dados fornecidos por outros membros da familia. As formas clinicas foram classificadas de acordo com Pessoa \& Barros, modificada por Barbosal.

A populaçào total das areas em estudo foi constituida por 492 individuos. sendo que 209 pertenciam à área de Menezes e 283 á área de Lagoa Redonda.

Todos os pacientes foram medicados com mebendazole na posologia de $200 \mathrm{mg} / \mathrm{dia} /$ três dias, com a finalidade de afastar a participaçào das outras helmintoses no quadro sintomatológico. Todos os individuos da área de Menezes, num total de 51 pessoas foram medicadas posteriormente com oxamniquine, constituindo-se no grupo caso e 51 individuos de Lagoa Redonda não receberam o esquistossomicida, constituindo-se no grupo controle. A oxamniquine foi prescrita na poso logia de $12,5 \mathrm{mg} / \mathrm{kg}$ a $15 \mathrm{mg} / \mathrm{kg}$ de peso corporal, nos pacientes com faixa etária igual ou superior aos quinze anos e de $15 \mathrm{mg} / \mathrm{kg}$ a $20 \mathrm{mg} / \mathrm{kg}$ de peso corporal para aqueles com idade compreendida entre nove a quatorze anos. No prazo mínimo de 45 dias pós-tratamento anti-esquistossomótico, colheu-se os sintomas vigentes. Em relação ao grupo controle, o levantamento dos sintomas foi feito no prazo mínimo de três semanas após o uso do mebendazole.

Os resultados foram analisados pelo teste de qui-quadrado em que a hipótese nula foi testada a um nivel de significância de 0,01 para $(\mathrm{r}-1)(\mathrm{s}-1)$ graus de liberdade.

\section{RESULTADOS}

A prevalência das helmintoses nos dois grupos inicialmente selecionados, pode ser observada na Tabela 1.

Tabela 1 - Prevalência das helmintoses em 73 individuos do grupo caso e 71 do grupo controle, na faixa etária de nove a dezenove anos, nas áreas de Menezes e Lagoa Redonda.

\begin{tabular}{lcccc}
\hline Helmintoses & $\begin{array}{c}\text { Grupo } \\
n .0\end{array}$ & $\begin{array}{c}\text { Caso } \\
\%\end{array}$ & $\begin{array}{c}\text { Grupo } \\
n .\end{array}$ & $\begin{array}{c}\text { Controle } \\
\%\end{array}$ \\
\hline S. mansoni & 51 & $(69,9)$ & 53 & $(74,6)$ \\
Ancilostomideos & 36 & $(49,3)$ & 34 & $(47,9)$ \\
A. lumbricoides & 44 & $(60,3)$ & 57 & $(80,3)$ \\
T. trichiuris & 22 & $(30,1)$ & 23 & $(32,4)$ \\
\hline
\end{tabular}

Tabela 2 - Distribuição das formas clínicas da esquistossomose entre os 102 indivíduos examinados nas áreas estudadas - $\mathrm{Me}^{-}$ nezes e Lagoa Redonda.

\begin{tabular}{lrr}
\hline Formas Clínicas & N.o & $\%$ \\
\hline Intestinal & 60 & 58,9 \\
Hepatointestinal & 38 & 37,2 \\
Hepatoesplênica & 4 & 3,9 \\
\hline Total & 102 & 100,0 \\
\hline
\end{tabular}

A freqüência das formas clinicas da esquistossomose é mostrada na Tabela 2, onde se observa que apenas quatro indivíduos apresentavam a forma hepatoesplênica da doença, três dos quais pertencentes ao grupo caso. Os sintomas intestinais nos grupos caso e controle são analisados na Tabela 3 , antes e depois da terapêutica com o mebendazole. A análise dos resultados constatou em ambos os grupos, diferenças estatisticamente significantes a nivel de 0,01 em relaçào à dor abdominal e diarréria. Os demais sintomas estudados não apresentaram diferenças significantes. A Tabela 4 apresenta os sintomas intestinais entre os dois grupos, 45 dias depois que o grupo caso foi tratado especificamente com oxamniquine. A analise estatistica demonstra melhora significativa das manifestações de diarréria, e de presença de muco e sangue nas fezes. 
Peixinho EL, André SB, Bina JC. Sintomatologia intestinal na fase crónica da esquistossomose mansoni. Revista da Sociedade Brasileira de Medicina Tropical 19: 27-30, Jan-Mar, 1986

Tabela 3 -Distribuição dos sintomas intestinais nos grupos caso e controle antes e depois da terapêutica como mebendazole.

\begin{tabular}{|c|c|c|c|c|c|c|c|c|}
\hline \multirow[t]{3}{*}{ Sintomas } & \multicolumn{2}{|l|}{ Grupo } & \multicolumn{2}{|l|}{ Caso } & \multicolumn{2}{|l|}{ Grupo } & \multicolumn{2}{|l|}{ Controle } \\
\hline & Antes & & Depois & & Antes & & Depois & \\
\hline & $N .^{\circ}$ & $\%$ & $N o$ & $\%$ & $N o$ & $\%$ & $N . o$ & $\%$ \\
\hline Dor abdominal & 33 & $(64,7)$ & 13 & $(25,5)$ & 31 & $(60,8)$ & 13 & $(25,5)$ \\
\hline Diarrẻia & 38 & $(74,5)$ & 25 & $(49,0)$ & 31 & $(60,8)$ & 15 & $(29,4)$ \\
\hline Fezes com muco & 30 & $(58,8)$ & 24 & $(47,0)$ & 30 & $(58,8)$ & 24 & $(47,0)$ \\
\hline Fezes com sangue & 28 & $(54,9)$ & 24 & $(47,0)$ & 29 & $(56,9)$ & 24 & $(47,0)$ \\
\hline Enterorragia & 0 & $(0,0)$ & 0 & $(0,0)$ & 1 & $(1,9)$ & 0 & $(0,0)$ \\
\hline
\end{tabular}

$\mathrm{X}_{1}^{2}=15,838 ; \mathrm{p}<0,01$ (dor abdominal)

$\mathrm{X}_{1}^{2}=7,015 ; \mathrm{p}<0,01$ (diarréia)

Tabela 4 - Distribuição dos sintomas intestinais entre o grupo caso-tratado com oxamniquine-e o grupo controle.

\begin{tabular}{llllll}
\hline Sintomas & Grupo & Caso & & Grupo & Controle \\
\cline { 2 - 3 } \cline { 5 - 6 } & N.o & $\%$ & & & No \\
\hline Dor abdominal & 7 & $(13,7)$ & 13 & $\%$ \\
Diarreia & 7 & $(13,7)$ & & 15 & $(25,5)$ \\
Fezes com muco & 6 & $(11,8)$ & 24 & $(47,0)$ \\
Fezes com sangue & 7 & $(13,7)$ & 24 & $(47,0)$ \\
Enterorragia & 0 & $(0,0)$ & 0 & $(0,0)$ \\
\hline
\end{tabular}

$\mathrm{X}_{1}^{2}=14.735: \mathrm{p} \leq 0,01$ (diarréia)

$\mathrm{X}_{1}^{2}=15,300 ; \mathrm{p}<0,01$ (presença de muco nas fezes)

$\mathrm{X}_{1}^{2}=13,393 ; \mathrm{p}<0,01$ (presença de estrias sangüineas nas fezes)

\section{DISCUSSÃO}

Os conhecimentos sobre os sintomas intestinais na fase crônica da esquistossomose mansoni são escassos e geralmente extraidos de amostras não isentas de outras condiçóes patologicas que podem interferir no quadro sintomático 378912 .

A metodologia utilizada no presente estudo limitou-se a pesquisar cinco sintomas intestinais, desprezando-se a existência de sintomas digestivos altos tais como pirose, eructações, vômitos, náuseas e empachamento pós-prandial, visto que seria indispensável descartar a participação da estrongiloidíase e outras manifestaçòes localizadas a nivel de esôfago e estômago, além da dificuldade na coleta desses sintomas, face ao precário grau das informações obtidas dos pacientes.
A população total das duas áreas estudadas apresenta alta prevalência de helmintos, fato este comum em toda região do Recôncavo Baiano. A prevalência da esquistossomose nos individuos da faixa etária determinada, inicialmente selecionados, medida por um único exame de fezes, foi bastante elevada e se equivaliam entre si (Tabela 1).

As áreas estudadas não apresentavam indivíduos intensamente parasitados, uma vez que somente cerca de $25 \%$ da população total eliminava mais de 500 ovos por grama de fezes. A quase totalidade dos individuos apresentava a forma intestinal ou hepatointestinal da esquistossomose, pois apenas quatro pacientes apresentavam a forma hepatoesplênica da doença - três no grupo caso e um no controle. Estes achados estão de acordo com a conclusão de outros 
Peixinho EL, André SB, Bina JC. Sintomatologia intestinal na fase crônica da esquistossomose mansoni. Revista da Sociedade Brasileira de Medicina Tropical 19: 27-30, Jan-Mar, 1986

autores, quando admitem que a gravidade da doença seria função da alta carga parasitária2 5101113 .

A prevalência de sintomas intestinais nos grupos caso e controle foram similares antes do tratamento com o mebendazole e a resposta de ambos os grupos ao tratamento das helmintoses intestinais foi a mesma. Assim, dor abdominal e diarréia diminuiram significativamente, permanecendo inalterado os sintomas de muco e sangue nas fezes. Entretanto, quando o grupo caso foi submetido à terapêutica anti-esquistossomótica, houve redução significante no tocante aos episódios diarréicos e principalmente quanto à presença de muco e sangue nas fezes (Tabelas 3 e 4 ).

$O$ valor da enterorragia como manifestação causada pelo $S$. mansoni, não pòde ser avaliado por ser de ocorrência praticamente nula. Embora discordantes dos trabalhos de Conceição ${ }^{3}$ e Menezes ${ }^{7}$, julgamos que provavelmente os nossos resultados decorram do pequeno numero de individuos intensamente parasitados.

Os resultados do presente trabalho sugerem que o sintoma dor abdominal decorra provavelmente da participação de outras helmintoses; os episódios diarreicos podem ser atribuidos tanto ao $S$. mansoni como às outras helmintoses; a presença de muco e sangue nas fezes entretanto, é bastante sugestiva do parasitismo pelo $S$. mansoni. Embora amebiase intestinal não tenha sido descartada no presente estudo, a resposta à terapêutica pela oxamniquine sugere que os sintomas dependiam da presença do $S$. mansoni.

\section{SUMMARY}

A total of 102 autochthonous patients from the districts of Lagoa Redonda and Menezes (Sapeaçu, $B$ Bhia), with ages ranging from nine to 19 years, were evaluated for intestinal symptoms related to the chronic phase of schistosomiasis mansoni, with emphasis on the following complaints: abdominal pain, diarrhea, mucus and blood in the stools. One half of the sample (case group) was treated with mebendazole followed by oxamniquine; the other half (control group) received only mebendazole. The results showed that schistosomiasis is probably responsible for the appearance of mucus and/or blood in the stools, since a statistically significant reduction of such manifestations was achieved with specific treatment.
Key words: Schistosoma mansoni. Schistosomiasis. Chronic phase of schistosomiasis. Intestinal manifestations.

\section{REFERÊNCIAS BIBLIOGRÁFICAS}

1. Barbosa FS. Morbidade da esquistossomose. Revista Brasileira de Malariologia e Doenças Tropicais: 3-159, 1966.

2. Cheever AW. A quantitative post-mortem study of Schistosomiasis mansoni in man. American Journal of Tropical Medicine and Hygiene 17:38-60, 1968.

3. Conceição MJ. Morbidade da esquistossomose mansônica em uma comunidade rural de Minas Gerais. Tese de Mestrado, Universidade Federal do Rio de Janeiro, Rio de Janeiro, 1976.

4. Katz N, Chaves A, Pellegrino J. A simple device for quantitative stool thick-smear technique in schistosomiasis mansoni. Revista do Instituto de Medicina Tropical de São Paulo 14:397-400. 1972.

5 . Kloetzel K. Splenomegaly in schistosomiasis mansoni. American Journal of Tropical Medicine and Hygiene 11:472-476, 1972.

6. Lutz A. O Schistosoma e a schistosomatose segundo observações feitas no Brasil. Memórias do Instituto Oswaldo Cruz 11:121-150, 1919.

7. Menezes AP. Esquistossomose mansônica no município de Riachuelo, Sergipe. (Estudo clinico, epidemiológico e laboratorial). Tese de Mestrado, Universidade Federal do Rio de Janeiro, Rio de Janeiro, 1976.

8. Neves J, Cunha AS. Esquistossomose mansoni. In: Neves J. (ed.) Diagnóstico e tratamento das doenças Infectuosas e Parasitárias. Guanabara-Koogan, Rio de Janeiro, p. 758-789, 1978.

9. Neves J, Santiago JM, Ratton JLA. Quadro clinico. In: Cunha AS (ed.) Esquistossomose Mansoni. Savier/Editora da Universidade de Sào Paulo, p. 131-191, 1970.

10. Pessoa SB, Amorim JP. Contribuição para a história natural da esquistossomose mansônica no nordeste brasileiro e sugestões para a sua profilaxia. Revista Brasileira de Malariologia e Doenças Tropicais 9:5-18, 1957.

11. Pessoa SB, Coutinho JO. A esquistossomose mansoni como doença do trabalho. O Hospital 43:429-436, 1953.

12. Prata A. Esquistossomose Mansoni. In: Veronesi R. (ed.) Doenças Infecciosas e Parasitárias. (7 a ed.) GuanabaraKoogan, Rio de Janeiro, p. 884-904, 1982.

13. Prata A., Bina JC. Development of the hepatosplenic form of schistosomiasis. A study of 20 patients observed during a 5 years period. Gazeta Medica da Bahia: 68 : 49-60. 1968. 